\title{
Radioprotection and Antitumor Effect by Lyophyllum decastes Singer and Propolis in Mice
}

\section{Yeunhwa Gu, Yuichi Ukawa, Masami Oshima, Ikukatsu Suzuki, Toshibiro Maenaka, Toshibiro Maenaka, E̊ In-Suk Choi}

Faculty of Health Science, Suzuka University of Medical Science, 1001 Kishioka-cho Suzuka Mie 510-0293, Japan

In this study, we examined the radioprotective effects of treatment with Lyophyllum decastes (Tricholomataceae, higher Basidiomycetes) and Propolis. It would obviously be counterproductive if the antiradiation effects of these substances prevented the antitumor effects of the radiation. Therefore, we examined the effect of radioprotective agents on the growth of tumors in combination with radiation therapy. Thus, the aim of this study is to take the first step to apply $L$. decastes and Propolis as new radioprotective agents and to obtain data for the development of these agents in the future.

As a possible mechanism, a crude polysaccharide fraction (ATF) was prepared promoting the invasion of NK cells into tumor tissues and inhibiting the appearance of the $\mathrm{S}$ phase in mitotic cycles of tumor cells. Many chemotherapeutic drugs cause adverse reactions. Not only tumor cells, but also other proliferating normal cells are damaged, and the function of the intestine, kidney, spinal cord, and immunity are affected because these drugs act by inhibiting DNA synthesis. Therefore, in general, antitumor drugs with fewer adverse reactions are desired. If antitumor drugs with fewer adverse reactions exist, the point of action would not be mediated by effects on DNA and RNA, which are common to both tumor cell and normal cells and may be associated with some specific tumor cell function involved in the relationship between the host and tumor cells. For example, antitumor effects can be caused by activation of the immune system and actions on vascularization caused by tumor tissues. In this study, L.decastes alone did not show a marked antitumor effect. One possible reason is that $L$. decastes does not show direct antitumor effects like those of artepillin $\mathrm{C}$, which is present in Propolis, but acts secondarily from an immunological aspect.

After a topical 6 Gy irradiation (therapy), the $L$. decastes group showed a tendency to suppress the tumor growth, as compared with the group that was only irradiated. It is thought that $L$. decastes does not show a direct antitumor effect. Therefore, the radioprotective effect reduces the decrease in immune cells, and a secondary antitumor effect generates a slight difference, as compared with the group that was only irradiated.

Thus, in radiation therapy, $L$. decastes shows an antitumor effect caused by a radioprotective effect on the immune cells, in addition to the antitumor effect caused by irradiation. 